\title{
ПРИМЕНЕНИЕ ИНСТРУМЕНТОВ ИМИТАЦИОННОГО МОДЕЛИРОВАНИЯ ПРИ ОПТИМИЗАЦИИ БИЗНЕС-ПРОЦЕССОВ
}

(C) 2020 Миронова Александра Леонидовна

студент

Сибирский федеральный университет, Россия, Красноярск

(C) 2020 Гаврилюк Екатерина Юрьевна

студент

Сибирский федеральный университет, Россия, Красноярск

(c) 2020 Гуслякова Анастасия Владиславовна

студент

Сибирский федеральный университет, Россия, Красноярск

(C) 2020 Свалова Анна Сергеевна

студент

Сибирский федеральный университет, Россия, Красноярск

(C) 2020 Гончар Вероника Николаевна

студент

Сибирский федеральный университет, Россия, Красноярск

В статье рассматривается актуальность моделирования бизнес-процессов в целях оптимизации деятельности предприятия с учетом быстросменяемых требований динамично развивающейся экономики. Описаны основные цели применения моделирования бизнес-процессов, задачи, решаемые при помощи его инструментов, виды моделирования в соответствии с исследуемыми характеристиками процесса и эффективность использования имитации на примере дискретнособытийного моделирования бизнес-процесса оказания инженерно-технических услуг.

Ключевые слова: моделирование, бизнес-процессы, оптимизация, модель, имитация, предприятие.

В текущей ситуации быстро развивающейся экономики современным предприятиям необходимо постоянно улучшать свою профессиональную деятельность для увеличения конкурентоспособности их компании. Для этого требуется создание новых технологий и решений по ведению бизнеса, а также, конечно, применение новых, наиболее эффективных способов управления и организации предприятия.

Деятельность компании рассматривается как набор специфических бизнес-процессов взаимосвязанных мероприятий, потребляющих ресурсы предприятия и создающих на выходе результат в виде ценности для потребителя. Чем выше эта ценность и чем дешевле осуществляемый процесс, тем эффективнее функционирует предприятие.

Ключевыми причинами для оптимизации имеющихся бизнес-процессов являются: уменьшение затрат или времени на производственный цикл, изменение требований к деятельно- сти предприятия, создание системы управления качеством, реорганизация компании и другие.

На сегодняшний день моделирование бизнес-процессов является эффективным инструментом в рамках управления предприятием, оптимизации бизнес-процессов, прогнозирования и стандартизации деятельности. Данный подход к реорганизации позволяет генерировать конкретные бизнес-сценарии существующей деятельности на заданном временном отрезке и выявлять наиболее эффективные сочетания полученных показателей, что является крайне актуальным в связи с ограниченностью как материальных ресурсов, так и временных, поскольку проводить реальный эксперимент долго, дорого и рискованно.

Так как ключевой задачей моделирования бизнес-процесса можно назвать повышение качества его функционирования, то наиболее важным является наращивание ценности его выходных показателей и снижение стоимости и 
времени на выполнение.

Можно выделить следующие основные цели моделирования бизнес-процессов:

- Описать процесс, в частности, точно знать, что происходит в процессе от его начала до завершения, что позволяет детально изучить процесс и получить информацию, на базе которой можно будет повысить его эффективность.

- Нормировать процесс, то есть задать ему правила выполнения благодаря моделированию, тем самым достичь планируемой производительности, если следовать требованиям, правилам и руководящим указаниям, определенных в модели.

- Установить четкие связи между процессами и требованиями, установленными для них, выделить ключевые функции и не допустить их дублирования, сокращая при этом как трудовые, так и материальные ресурсы на их выполнение.

K достоинству моделирования бизнеспроцессов для анализа их эффективности можно отнести универсальность модели, т.к. в первую очередь, качественно составленная модель может дать ответ на практически все вопросы улучшения деятельности компании и повышения ее конкурентоспособности на рынке, при этом, внедряя полученную методологию, руководство будет иметь возможность самостоятельно менять и совершенствовать осуществляемые бизнес-процессы, прогнозируя развитие предприятия при помощи инструментов моделирования.

Комплексный подход к моделированию должен включать в себя несколько этапов работ с процессом, в том числе внедрение моделей, анализ полученных результатов, совершенствование имеющейся модели и другие. Рассмотрим следующие стадии моделирования бизнеспроцессов [1]:

- Выявление процессов и построение исходной модели «как есть»;

Дальнейшая оптимизация процесса невозможна без понимания, как компания функционирует сейчас. На данном этапе необходимо обозначить границы процесса, его внутренние и внешние составляющие, ключевые элементы и характеристики его работ, которые затем послужат основой модели «как есть».

- Пересмотр, анализ и уточнение исходной модели;

Анализ модели «как есть» выявляет противоречия и дублирование функций в процессе, что акцентирует внимание о необходимости изменений и создании окончательного варианта модели «как есть».

- Разработка модели «как должно быть»;

Имея актуальную, достоверную и проанализированную информацию о текущей ситуации, определяется усовершенствованное состояние бизнес-процесса, которого компания стремится достичь в своей деятельности. Данный эталон должен быть заложен в модель «как должно быть». Такой тип модели транслирует уже оптимизированный бизнес-процесс.

- Тестирование и применение модели «как должно быть»;

Следующим этапом является внедрение в бизнес-деятельность компании полученной «идеальной» модели ее осуществления. Модель «как должно быть» проходит апробацию на практике, при необходимости в нее вносятся коррективы.

- Улучшение модели «как должно быть».

Моделирование бизнес-процессов требует постоянной работы, после внедрения оптимизированной модели производства бизнеспроцессы продолжают меняться, и их новым, актуальным состояниям необходимы анализ и совершенствование, в связи с чем, необходимо регулярно пересматривать и улучшать деятельности компании, с чем и связан данный этап.

Моделирование бизнес-процессов может преследовать различные цели, их направленность зависит от того, какие задачи с его помощью планирует решить руководство предприятия. Желание рассмотреть все возможные проблемы может усложнить модель и снизить ее полезность избыточностью описания процессов. В соответствии с этим, выделяют различные виды моделирования в зависимости от характеристик процесса, которые планируется оптимизировать. Несмотря на это в отношении одного и того же процесса могут быть применены разные виды моделирования.

Для совершенствования процесса применяются:

- Функциональное моделирование;

Процесс рассматривается как совокупность имеющихся структурированных функций и их взаимосвязей.

- Объектное моделирование;

Процесс рассматривается как набор взаимодействующих объектов - т.е. производственных единиц. 
- Имитационное моделирование.

Данный вид предусматривает моделирование поведения процессов при воздействии различных внутренних и внешних факторах, с анализом изменяемых характеристик и распределения финансовых и временных ресурсов.

Далее на примере предприятия для того, чтобы выявить основные пути оптимизации его деятельности, будет использовано имитационное моделирование, которое позволяет бизнесаналитику использовать реальные данные для калибровки модели бизнес-процесса и выполнения разработки и тестирования альтернативных вариантов, и сценариев типа «что - если» [2], что в свою очередь предоставляет возможность предприятию избежать убытков от нерационального устройства своей деятельности.

Рассмотрим эффективность использования имитации на примере дискретно-событийного моделирования бизнес-процесса оказания инженерно-технических услуг. Данный вид имитационного моделирования представляет процесс как хронологическую последовательность событий во времени.

Перед руководством стоит задача по оптимизации существующего процесса путем использования электронной цифровой подписи, которая упрощает процесс документооборота, сокращая при этом издержки на обработку и передачу документов. Для этого проведем эксперимент путем взаимодействия модели бизнеспроцесса с моделью внешней среды и выявим основные пути оптимизации. Используя имитационное моделирование в качестве апробирова- ния существующих гипотез по рационализации, предприятию удастся избежать лишних расходов, связанных с неудачным переустройством реального бизнеса.

Используется модель рассматриваемого бизнес-процесса и модель внешней среды платформа сервиса bpsimulator.com посредством внедрения в модели внешних объектов - Генераторов задач. Платформа имитирует наступление определенного времени в реальном мире и рассчитывает состояние бизнес-процесса в этот временной срез [3].

Входными параметрами для данной симуляции был выбраны:

- количество заданных задач - 10;

- время работы - с 9 до 17 часов при равномерном распределении;

- длительность каждой функции - 3 часа;

- количество сотрудников по каждой должности - 1 .

Также для каждого исполнителя была назначена стоимость выполнения работы в час (условное значение) и время работы (соответствует общему времени).

Результат симуляции представлен на рисунке 1.

Как видно из рисунка, выработка бизнеспроцесса составляет только 75\% из возможных 100, при созданных 10 задачах за весь рабочий день выполняется только 6, при этом стоимость цикла бизнес-процесса составляет порядка 120 тысяч рублей. В связи с чем, необходимо провести оптимизацию процесса и заново провести симуляцию.

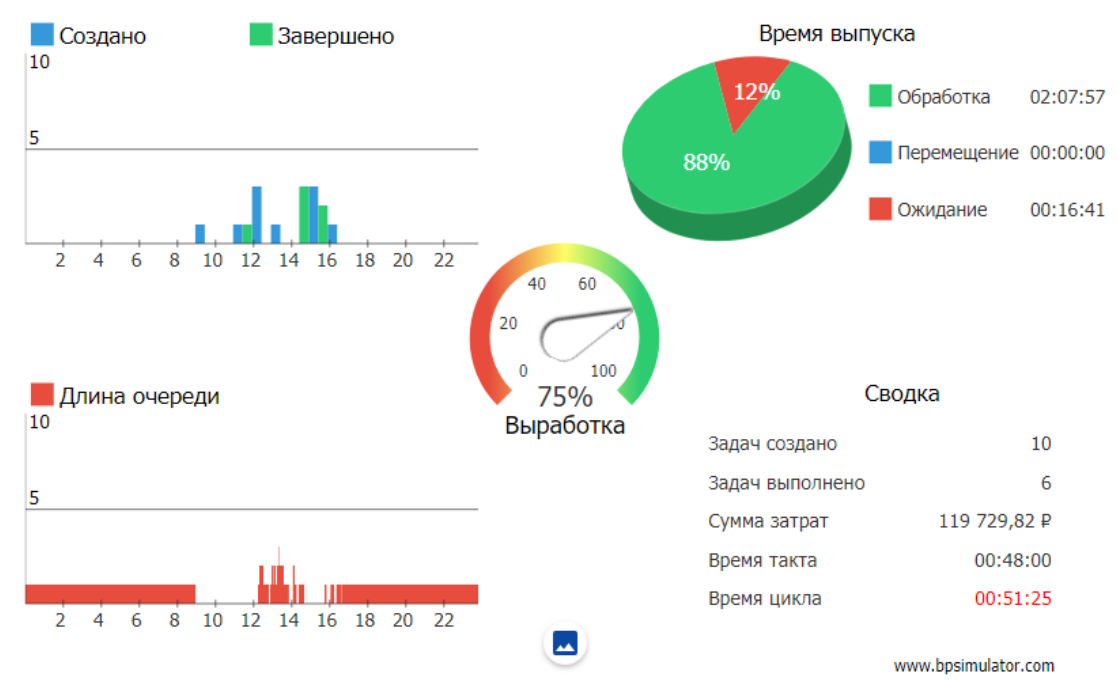

Рисунок 1. Результаты симуляции 
На основании результатов, полученных на предыдущем этапе, были выбраны следующие критерии, нуждающиеся в оптимизации: производительность, время ожидания обработки и загруженность исполнителей.

Добиться увеличения производительности, снижения времени ожидания и загруженности исполнителей попробуем за счет использования электронной цифровой подписи.

Полученный результат представлен на рисунке 2.

Как видно из рисунка, оптимизация прошла успешно. За счет использования электронной подписи сократилось время на выполнение каждой задачи, следовательно, время цикла теперь занимает меньше времени, как и ожидание с обработкой. Стоимость затрат увеличилась незначительно из-за оформления электронной подписи, но производительность теперь соответствует $100 \%$.

В процессе анализа значения моделируемых показателей могут оказаться неудовлетворительными. Для того чтобы это исправить, можно изменить некоторые аспекты имеющейся модели в рамках реализуемой идеи оптимизации и провести имитацию вновь. После всех проведенных экспериментов можно выбрать те условия, которые обеспечивают наиболее эффективные результаты, и внедрить их в собственный бизнес.

Таким образом, на примере готового бизнес-процесса было проведено дискретнособытийное моделирование, результаты которого свидетельствуют о том, что инструмент имитационного моделирования с учетом внешних и внутренних факторов позволяет выявлять слабые места бизнес-процесса и обозначать основные пути их оптимизации. Изменение значений основных критериев модели процесса на альтернативные позволяет преобразовывать входы в выходы с большей производительностью и меньшими затратами. По результатам симуляции модели можно получить важную информацию по оптимизации процесса для эффективного управления предприятием, совершенствования и прогнозирования различных вариантов его реорганизации, избежав при этом возможных расходов при проведении реального эксперимента.

Имитационное моделирование полезно не только для существующего предприятия, но и для будущего - при помощи инструментов моделирования можно спроектировать новый бизнес, ответив на вопросы будущей организационной структуры, частоты выполнения бизнес-процессов, их оптимальных продолжительности и стоимости. Провести имитацию будущего бизнеса оказывается гораздо дешевле и менее рискованно, чем воплощать расплывчатые идеи на реальных работниках и материальных ресурсах. В интересах компании ответить на все эти вопросы до того, как начнется реальное производство, в процессе которого выяснится, что трудовые ресурсы перегружены, а материальные находятся в дефиците. Поэтому если рационально смоделировать эффективно работающий бизнес сразу, то многие проблемы

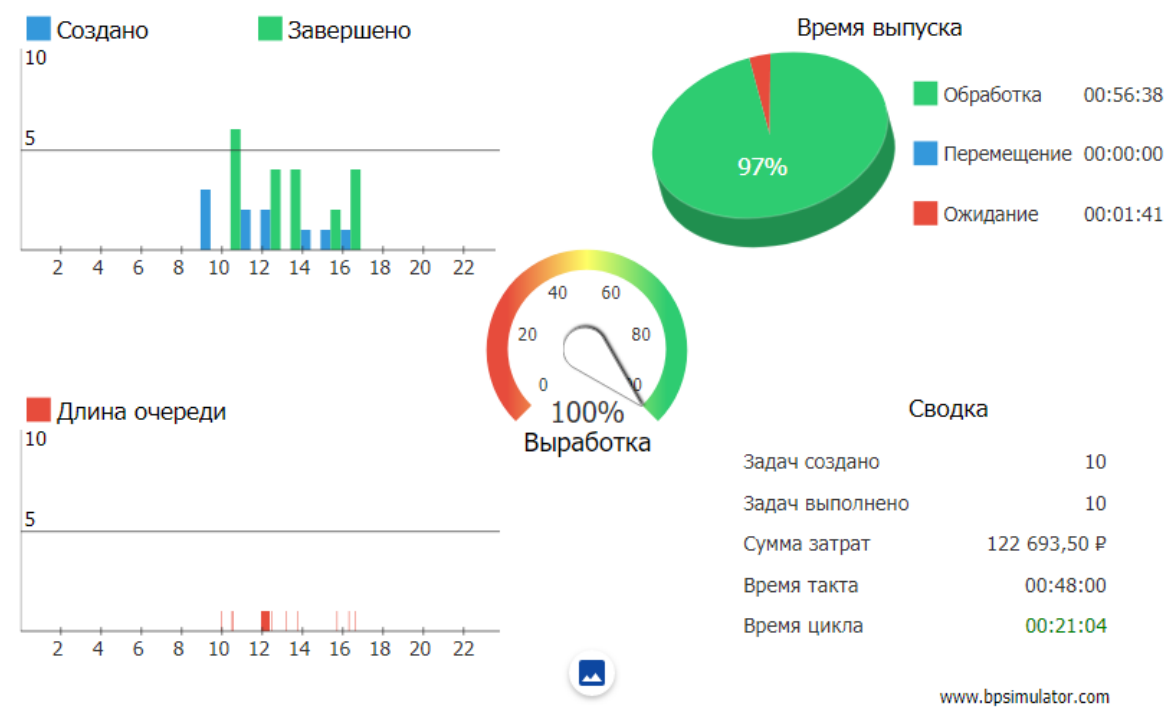

Рисунок 2. Симуляция бизнес-процесса после оптимизации 
просто не возникнут, что сэкономит ценное время и финансы, которых у молодой компании и так крайне ограниченное количество.

Можно сделать вывод, что, понимая бизнеспроцесс как набор действий, который выполняется на предприятии для получения заданного результата, а всю деятельность предприятия, рассматривая как совокупность некоторого количества бизнес-процессов, бизнес-моделирование позволяет избежать чрезмерного разрастания численности персонала, снижает вероятность внутренней конкуренции между подразде- лениями организации, а также может стать эффективной базой для совершенствования деятельности компании, т.к. предоставляет возможность визуализировать, по сравнению с текстовым форматом, результаты моделирования - финансовые и временные потери, лишние операции, простои в работе сотрудников, избыточные должности и функции. Таким образом, моделирование позволяет более точно анализировать и принимать управленческие и организационные решения.

\section{Библиографический список}

1. Автоматизация СМК. Моделирование бизнес процессов [Электронный ресурc]. URL: https://www.kpms.ru/ Automatization/BPM.htm [дата обращения: 30.09.2020].

2. НИУ ВШЭ, 2008: Бизнес-аналитика: создание бизнес-процесса с помощью инструментов Rational и WebSphere [Электронный ресурс]. URL: https://www.intuit.ru/studies/professional_retraining/19206/ courses/258/info [дата обращения: 30.09.2020].

3. Вьюненко Л. Ф. Имитационное моделирование [Электронный ресурс].URL: http://bookash.pro/ru/book/85589/ imitatsionnoe-modelirovanie-uchebnik-i-praktikum-dlya-akademicheskogo-bakalavriata-lyudmila-fedorovn

4. БП-симулятор, 2019: Справочное руководство [Электронный ресурс]. URL: https://www.bpsimulator.com/ru/ help/simulation.html [дата обращения: 16.10.2020].

5. Иноземцев, В.Л. Парадоксы постиндустриальной экономики [Текст] / В.Л.Иноземцев // Мировая экономика и международные отношения.-2000.- № 3.- С. 3-11 